\title{
Presiones inspiratoria y espiratoria máximas: Recomendaciones y procedimiento
}

\section{Maximal inspiratory and expiratory pressures: Recommendations and procedure}

\author{
Uri de Jesús Mora-Romero, * Laura Gochicoa-Rangel,* Selene Guerrero-Zúñiga,* Silvia Cid-Juárez,* \\ Mónica Silva-Cerón,* Isabel Salas-Escamilla, ${ }^{*}$ Luis Torre-Bouscoulet*
}

*Instituto Nacional de Enfermedades Respiratorias Ismael Cosío Villegas, Ciudad de México.

\begin{abstract}
RESUMEN. La medición de las presiones inspiratoria $\left(\mathrm{Pi}_{\max }\right)$ y espiratoria $\left(\mathrm{Pe}_{\max }\right)$ máximas permite evaluar la fuerza de los músculos respiratorios. Son mediciones sencillas, rápidas y no invasivas. Consisten en que el paciente debe generar la máxima presión inspiratoria (a partir de volumen residual) y espiratoria (a partir de capacidad pulmonar total) contra un sistema ocluido. La $\mathrm{Pi}$ evalúa principalmente la fuerza diafragmática; mientras que $\mathrm{Pe}_{\max ^{\prime}}$ la de los músculos intercostales y abdominales. Las presiones respiratorias máximas (PRM) resultan de gran utilidad clínica en el diagnóstico y seguimiento de enfermedades que afectan a los músculos respiratorios. Con el objetivo de mejorar la calidad y facilitar la realización de la medición de las PRM, revisamos las recomendaciones internacionales en materia de este procedimiento y emitimos lineamientos locales que contribuyen a un mejor proceso de estandarización.
\end{abstract}

Palabras clave: Presión inspiratoria máxima, presión espiratoria máxima, función pulmonar, procedimiento, fuerza muscular, músculos respiratorios, diafragma.

\section{INTRODUCCIÓN}

La medición de las presiones inspiratoria $\left(\mathrm{Pi}_{\max }\right)$ y espiratoria $\left(\mathrm{Pe}_{\max }\right)$ máximas permite evaluar la fuerza de los músculos respiratorios. $\mathrm{La} \mathrm{Pi}_{\max }$ evalúa principalmente la fuerza diafragmática; mientras que la $\mathrm{Pe}_{\text {max }^{\prime}}$ la de los músculos intercostales y abdominales. La medición de las presiones respiratorias máximas (PRM) es sencilla y consiste en que el paciente debe generar la máxima presión inspiratoria (a partir de volumen residual) y espiratoria (a partir de capacidad pulmonar total) contra una vía o equipo ocluido. ${ }^{1-3}$

\section{Correspondencia:}

Dra. Laura Gochicoa-Rangel

Jefa del Departamento de Fisiología Respiratoria, Instituto Nacional de Enfermedades Respiratorias Ismael Cosío Villegas.

Calzada de Tlalpan Núm. 4502, Colonia Sección XVI. 14080 Ciudad de México. Teléfono: +52 -55 -54871700 extensión 5160

Correo electrónico: gochis@dr.com

Trabajo recibido: 24-X-2014; aceptado: 27-X-2014.
ABSTRACT. The measurement of maximal inspiratory (MIP) and expiratory (MEP) pressures assess the strength of the respiratory muscles. These test are a simple, quick, and noninvasive. It consists that the patient must generate peak inspiratory (from residual volume) and expiratory pressure (from total lung capacity) against an occluded system. MIP assesses the diaphragmatic strength; while MEP assess the intercostal and abdominal muscles. Maximum respiratory pressures are of great clinical utility in the diagnosis and monitoring of diseases affecting the respiratory muscles. In order to improve quality and facilitate the realization of the measurement of MIP-MEP, we review international recommendations on this procedure and issue local guidelines that contribute to a better standardization process.

Keywords: Maximal inspiratory pressure, maximal expiratory pressure, maximal respiratory pressures lung function, procedure, muscle strength, respiratory muscles, diaphragm.

Con el objetivo de mejorar la calidad y facilitar la realización de la medición de las PRM, revisamos las recomendaciones internacionales ${ }^{2,3}$ en materia de este procedimiento y emitimos lineamientos locales que contribuyen a un mejor proceso de estandarización. Este documento forma parte de una serie de Recomendaciones y Procedimientos para diversas pruebas de función respiratoria que NCT ha publicado en números previos. ${ }^{4-6}$ Se mencionan las indicaciones y contraindicaciones, los recursos y características con las que debe contar un laboratorio de función respiratoria para su ejecución, la preparación e indicaciones para el paciente y describimos detalladamente el procedimiento así como las directrices para su interpretación.

\section{INDICACIONES Y CONTRAINDICACIONES}

La medición de las PRM no debe realizarse con fines de escrutinio. Con mucho, la indicación más importante es en aquellas enfermedades que en algún momento de su evolución, cursan con debilidad de los músculos de la res- 
Tabla 1: Indicaciones de la medición de presiones respiratorias máximas. 2,3,7

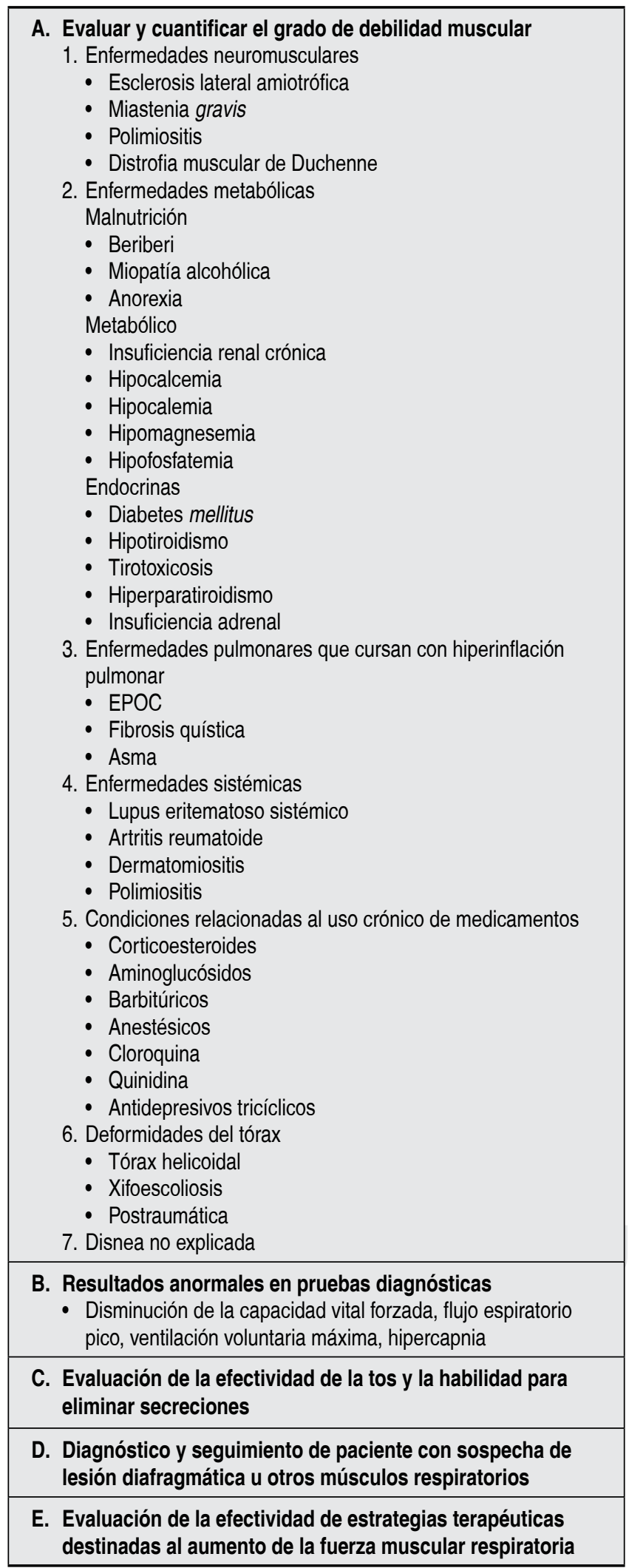

piración. En las Tablas 1 y 2 se enumeran las indicaciones y contraindicaciones de esta prueba.

\section{RECURSOS MATERIALES}

1. Equipo de medición de las PRM: De acuerdo a las recomendaciones ATS/ERS 2002, el equipo para realizar las mediciones de $\mathrm{Pi}_{\max }-\mathrm{Pe}_{\max }$ debe contar con un transductor de presión tipo piezo-eléctrico, con una precisión de $0.049 \mathrm{kPa}\left(0.5 \mathrm{cmH}_{2} \mathrm{O}\right)$ y en un rango de presión de $\pm 19.6 \mathrm{kPa}\left( \pm 200 \mathrm{cmH}_{2} \mathrm{O}\right)$ (Figura 1). El sistema requiere una pequeña fuga (aproximadamente de $2 \mathrm{~mm}$ de diámetro interno [DI] y de $20-30 \mathrm{~mm}$ de longitud) para evitar el cierre de la glotis durante la maniobra de $\mathrm{Pi}_{\max }$ y para reducir el uso de los músculos de la boca durante la maniobra de $\mathrm{Pe}_{\text {max }}$ (Figura 2).

2. El equipo debe contar con un sistema de registro para recoger los datos de presión de la maniobra y mostrarlo en forma analógica (tira de registro gráfico), o puede ser digitalizado y mostrar el promedio de la medición durante una meseta de al menos un segundo.

3. Boquillas tipo buceo: Ha sido explorado el efecto del uso de los distintos tipos de boquillas (piezas bucales) en la estimación de las $\mathrm{PRM}^{8,9}$ (boquilla rígida vs. boquilla tipo buceo). Las recomendaciones internacionales2,3 indican que el tipo de boquilla que debe utilizarse es de tipo buceo, debido a que éstas son de uso común, confieren mayor comodidad al paciente y podrían mejorar la coordinación para realizar las maniobras (Figura 3).

4. Filtros para conexión de la boquilla con el equipo.

5. Pinza nasal (no es estrictamente necesaria).

6. Báscula, estadímetro, baumanómetro, carro de paro.

7. Impresora.

8. Aditamentos para control de infecciones.

Tabla 2: Contraindicaciones de la medición de las presiones respiratorias máximas.

\section{A. Contraindicaciones absolutas}

1. Angina inestable

2. Infarto de miocardio reciente (4 semanas siguientes al evento) o miocarditis

3. Hipertensión arterial sistémica no controlada

4. Neumotórax reciente

5. Posoperatorio de biopsia pulmonar (una semana)

6. Posoperatorio de cirugía abdominal o genitorurinaria

7. Incontinencia urinaria

\section{B. Contraindicaciones relativas}

1. Presión arterial diastólica en reposo $>110 \mathrm{mmHg}$ o presión arterial sistólica en reposo $>200 \mathrm{mmHg}$

2. Lesión espinal reciente

3. Lesión ocular reciente

4. Pacientes poco colaboradores o incapaces de realizar la prueba por debilidad, dolor, fiebre, disnea, falta de coordinación o psicosis 


\section{INSTRUCCIONES PARA EL PACIENTE ANTES DE LA PRUEBA}

Cuando el paciente acude a solicitar cita para el estudio, se le deben entregar por escrito las siguientes indicaciones para la prueba:
1. Acudir al laboratorio vistiendo ropa cómoda, preferentemente deportiva.

2. No acudir con prendas restrictivas de tórax o abdomen, como chalecos, corsés o ropa muy ajustada.

3. Haber consumido un desayuno ligero.

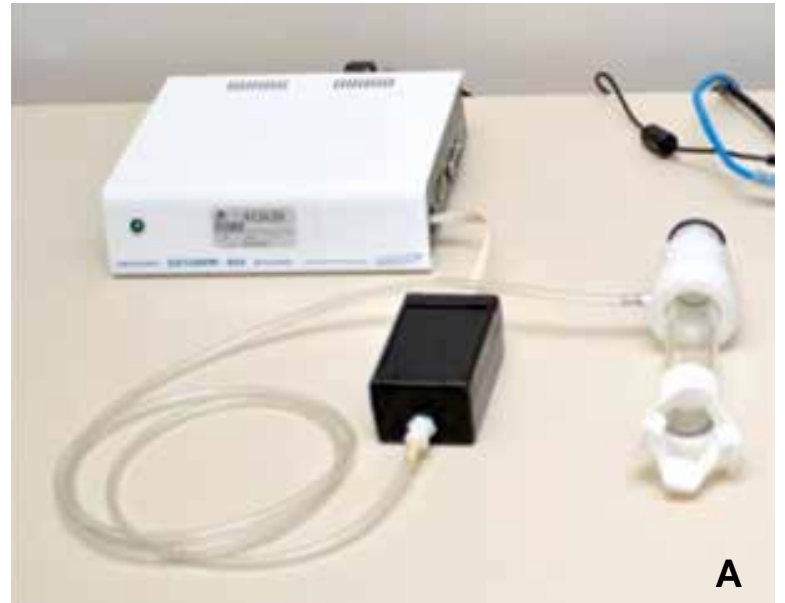

A

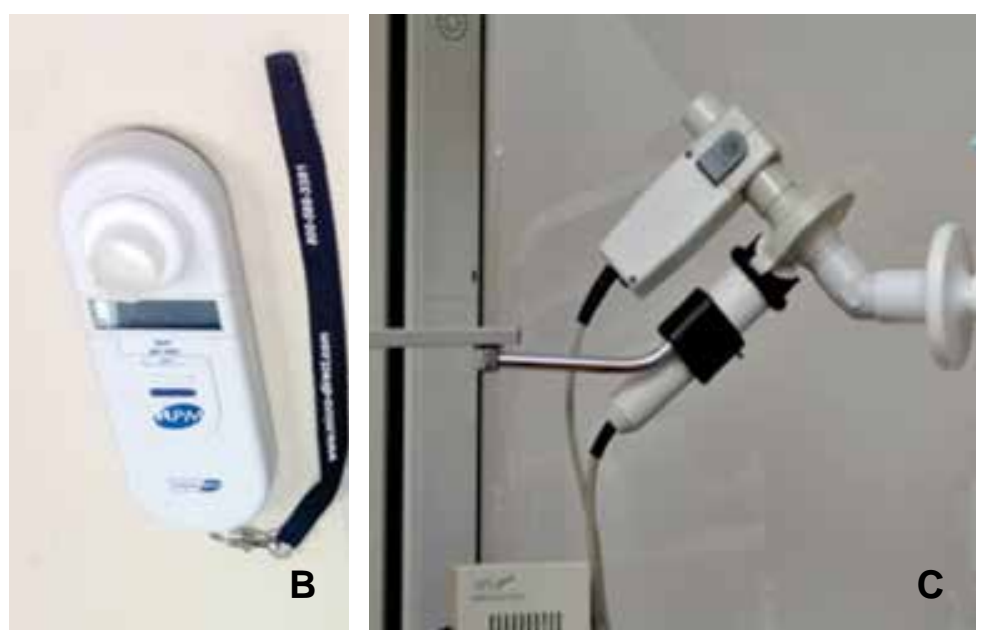

Figura 1:

Diferentes equipos para medir $\mathrm{Pi}_{\max }-\mathrm{Pe}_{\max }$.

A) Equipo Sibelmed DATOSPIR-120 PIM-PEM ${ }^{\circledast}$,

B) Carefusion MicroRPM ${ }^{\oplus}$

C) MasterScreen PFT Jaeger ${ }^{\circledR}$ y

D) Paul-Enright ${ }^{\ominus}$.

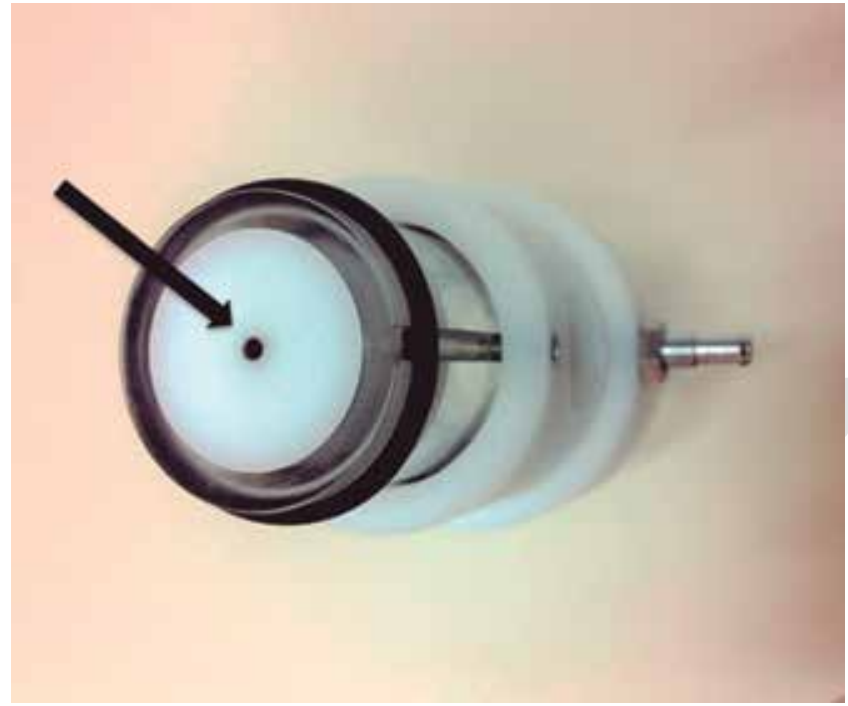

Figura 2: La flecha muestra la fuga que debe presentar el equipo para evitar cierre glótico durante la maniobra de $\mathrm{Pi}_{\text {max }}$ y el uso excesivo de los músculos de la boca durante la maniobra de $\mathrm{Pe}_{\max }$.
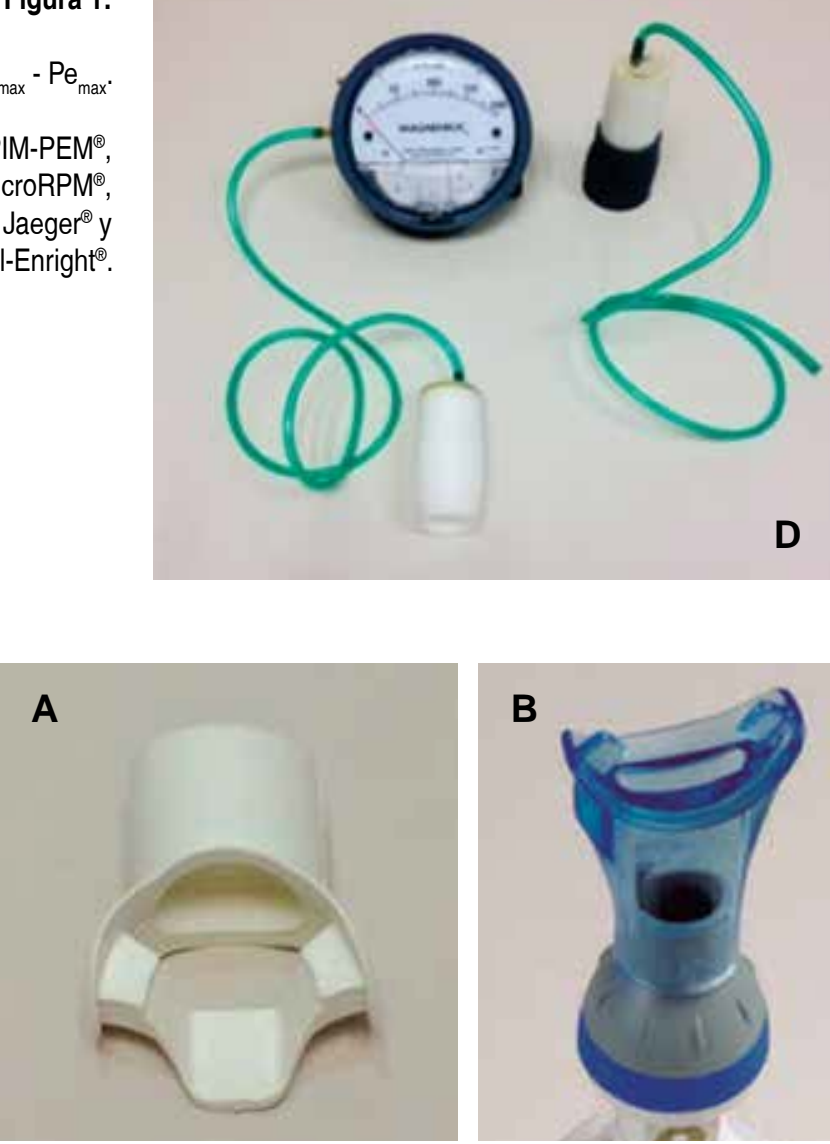

Figura 3: A) Boquilla tipo buceo. B) Boquilla colocada a filtro. 
4. No realizar ejercicio vigoroso cuatro horas previas al estudio.

5. Evitar tabaquismo al menos dos horas antes de la prueba.

6. No es necesario interrumpir la medicación habitual del paciente antes del examen.

\section{Preparación del equipo y del laboratorio de función pulmonar antes de la prueba}

1. Los equipos deben estar desinfectados y calibrados de acuerdo a las consideraciones del fabricante y verificar el buen funcionamiento de los mismos.

2. Se debe verificar la calibración de los transductores de presión contra un manómetro (línea de base igual a la presión atmosférica). En la Figura 4 observamos la calibración de un equipo portátil con un manómetro graduado en centímetros de agua $\left(\mathrm{CmH}_{2} \mathrm{O}\right)$.

\section{Preparación del paciente para la prueba}

1. El técnico que realiza la prueba se presenta con el paciente y revisa la solicitud.

2. Verifica el nombre completo y fecha de nacimiento del paciente así como el número de identificación.

3. Revisa que no existan contraindicaciones para la prueba y en caso de identificar alguna, deberá informar al personal médico responsable del laboratorio.

4. Se cerciora que el paciente haya acudido de acuerdo a las recomendaciones; y en caso contrario, informar al personal médico del laboratorio.

5. El técnico explica al paciente el objetivo del procedimiento, la frase más sencilla recomendada es la siguiente: «La medición de las presiones respiratorias máximas consiste en meter y sacar el aire con toda la fuerza posible, con lo que podremos conocer la fuerza de los músculos que utilizamos para respirar. Realizaremos varias mediciones y cuando tengamos tres mediciones casi iguales habremos terminado. Si usted considera que ya no puede continuar, o tiene alguna molestia como mareo o dolor intenso en el pecho, le pedimos nos lo comunique para detener la prueba».

6. El técnico procede a medir, pesar y hacer el registro del paciente. La estatura se mide en centímetros y con el individuo sin zapatos, en posición completamente erguida, talones juntos y mirando al frente. Para la medición de estatura es recomendable el uso de estadímetros de pared. El peso se mide en una báscula calibrada y se registra en kilogramos en unidades cerradas al $0.5 \mathrm{~kg}$ más cercano. La edad se registra en años cumplidos al día de la prueba.

7. En los pacientes que no puedan mantenerse de pie o sufran de deformidad de caja torácica, se puede usar la extensión de los brazos como una estimación de la

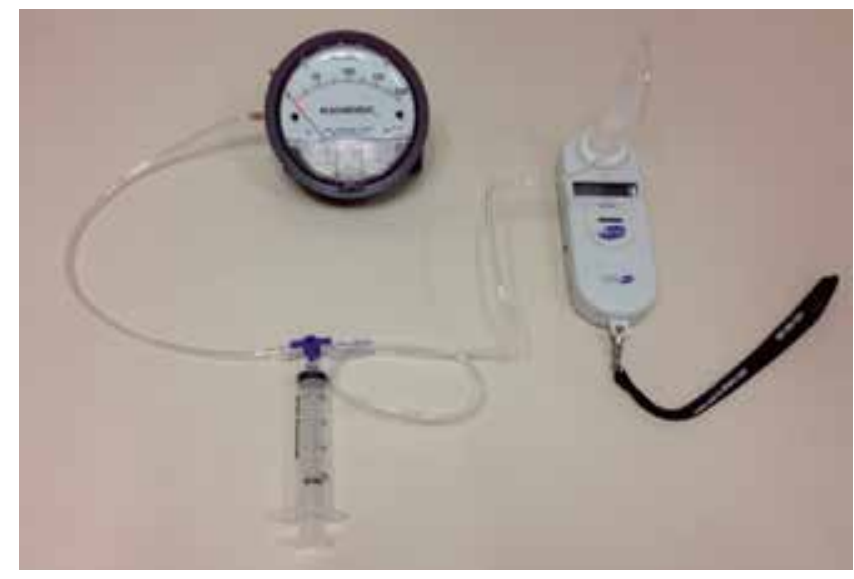

Figura 4: Calibración de presión de equipo portátil.

estatura. Se solicita al paciente que extienda al máximo los brazos en direcciones opuestas. Se mide la extensión entre el extremo de los dedos medios de cada mano. La estatura se estima para hombres como extensión de brazos en centímetros dividido entre 1.03 y para mujeres dividido entre 1.01.

Una vez registrados los datos del paciente y habiéndole explicado el objetivo se procede a la realización del estudio.

\section{PROCEDIMIENTO}

La prueba debe realizarse con el sujeto sentado. Se deben utilizar sillas sin ruedas y con soporte para los brazos. Se coloca al sujeto sentado con el tórax y cuello en posición erguida y con ambos pies apoyados sobre el piso. Si se utiliza una posición diferente, ésta debe de registrarse $\mathrm{e}^{2,3,10}$ ya que en pacientes con EPOC las mediciones obtenidas con inclinación hacia adelante, ${ }^{11}$ pueden ser mayores que las obtenidas en la posición en decúbito.?

Se explica el procedimiento y se demuestra la prueba en el siguiente orden:

1. El paciente permanecerá sentado durante la prueba (Figura 5).

2. Una persona o el mismo paciente, darán soporte a las mejillas, principalmente para la $\mathrm{Pe}_{\max }{ }^{2,3,12}$

3. Se le colocará una boquilla con filtro en la cual no debe introducir la lengua, ni morderla y tratará de sellar los labios alrededor de la misma.

4. Advierta al paciente que realizar esfuerzos respiratorios intensos puede molestar sus oídos; pero aún así, debe realizar un esfuerzo máximo.

5. Enfatice la necesidad de mantener los labios perfectamente cerrados para evitar fugas. 


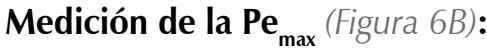

1. Solicite al paciente que exhale suave, pero completamente (con el fin de llegar a volumen residual) y que luego inhale tan fuerte y rápido como le sea posible. La duración de la presión máxima alcanzada debe ser idealmente de 1.5 segundos para poder obtener el promedio de medición durante un segundo.

2. El pico de presión puede ser más alto que la presión de un segundo sostenido, pero se considera que es menos reproducible por lo que se prefiere el promedio de la presión máxima durante un segundo (ATS-ERS 2002).

3. Estimule al paciente para que lo haga con toda la fuerza posible.

4. Ya que los resultados son considerablemente dependientes del esfuerzo del paciente, obtenga tres intentos reproducibles (menos de 10\% de diferencia entre los dos de mayor valor) de un máximo de ocho y mínimo de cinco intentos.

5. Permita que el paciente descanse 60 segundos entre un intento y otro.
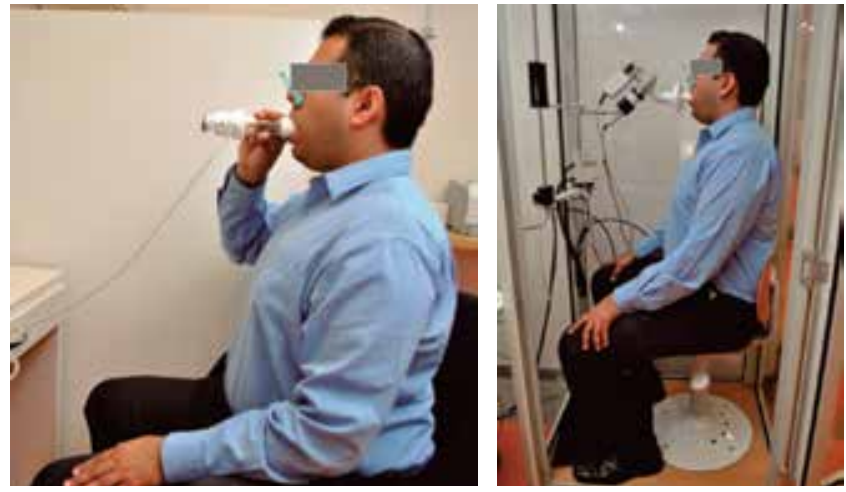

Figura 5: El paciente realiza la maniobra sentado en una silla sin ruedas, con los pies apoyados en el piso y con el cuerpo en posición erguida.
1. Solicite al paciente que inhale profundo y completamente (con el fin de llegar a capacidad pulmonar total) y luego que exhale tan fuerte y rápido como le sea posible.

2. Estimule al paciente para que lo haga con toda la fuerza y asegure que no se presenten fugas.

3. La duración de la presión máxima alcanzada debe ser idealmente de 1.5 segundos para poder obtener el promedio de medición durante un segundo.

4. Obtenga tres intentos reproducibles (menos del $10 \%$ de diferencia entre los dos de mayor valor) de un máximo de ocho. Si el último intento es el mayor de todos realice una nueva maniobra.

5. Permita que el paciente descanse 60 segundos entre un intento y otro.

\section{Complicaciones:}

1. Ruptura timpánica

2. Síncope

3. Hemorragia conjuntival

4. Cefalea

\section{INFORME DE LOS RESULTADOS (Figura 7)}

1. El informe debe incluir los datos demográficos de cada paciente.

2. Los resultados de los valores medidos para la $\mathrm{Pi}_{\text {max }} \mathrm{y}$ $\mathrm{Pe}_{\text {max }}$ se expresan usualmente en $\mathrm{CmH}_{2} \mathrm{O}$.

3. Se informará el mayor de tres intentos reproducibles teniendo en cuenta que se deben de eliminar los picos de presión registrados menores a un segundo de duración.

4. Los datos se presentan en valor absoluto y como porcentaje de predichos.

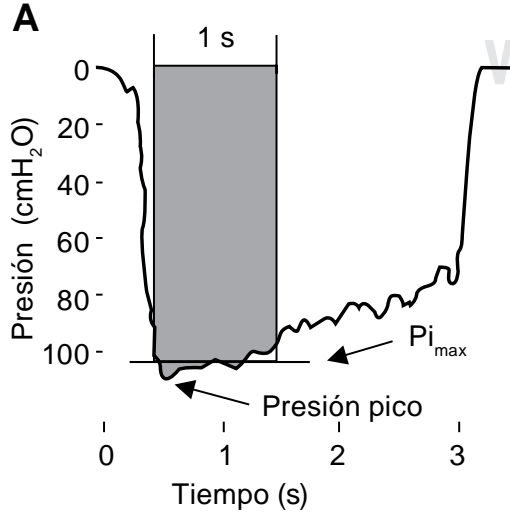

Tiempo (s)

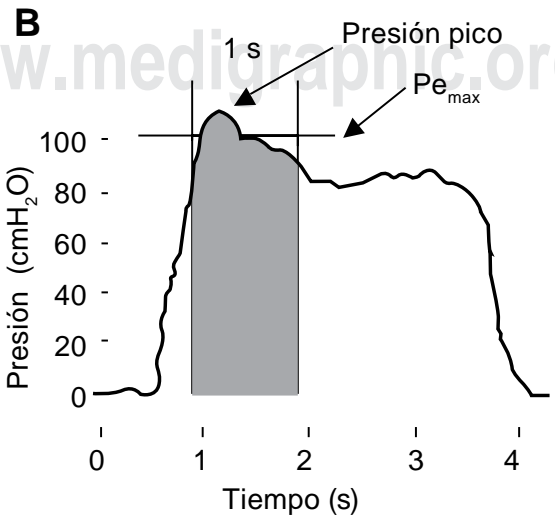

Figura 6:

A. Gráfica de la maniobra de $\mathrm{Pi}_{\text {max }}$

B. Gráfica de la maniobra de $P e_{\text {max }}$

Se observan las curvas de las maniobras de presiones respiratorias máximas, la presión pico y el área sombreada, ésta es utilizada para calcular el promedio de presión durante un segundo. 
Nombre de la institución Reporte de $\mathrm{Pi}_{\max }-\mathrm{Pe}_{\max }$

\begin{tabular}{|c|c|c|c|c|c|}
\hline Nombre: & CAST... ROD.. RA.. & Edad: & 27 años & $\begin{array}{l}\text { Fecha: } \\
\text { Hora: }\end{array}$ & $\begin{array}{l}\mathrm{AA} / \mathrm{MM} / \mathrm{DD} \\
09: 00 \mathrm{~h}\end{array}$ \\
\hline $\begin{array}{r}\text { Fecha de nacimiento: } \\
\text { Sexo: } \\
\text { Calibración: }\end{array}$ & $\begin{array}{l}\text { AA/MM/DD } \\
\text { Masculino } \\
\text { AA/MM/DD }\end{array}$ & $\begin{array}{l}\text { Peso: } \\
\text { IMC: }\end{array}$ & $\begin{array}{l}62 \mathrm{~kg} \\
21.0\end{array}$ & $\begin{array}{r}\text { Talla: } \\
\text { Predichos: }\end{array}$ & $\begin{array}{l}172 \mathrm{~cm} \\
\text { Black-Haytt, } 1969\end{array}$ \\
\hline
\end{tabular}

\begin{tabular}{lcccc} 
Maniobra & $\mathrm{Pi}_{\max }$ & $\mathrm{CmH}_{2} \mathrm{O}$ & $\mathrm{Pe}_{\max }$ & $\mathrm{CmH}_{2} \mathrm{O}$ \\
\hline M1 & 100 & & 97 & \\
M2 & 94 & & 99 & \\
M3 & 99 & & 98 & \\
M4 & 92 & & 96 & \\
Mejor maniobra & 100 & $\mathrm{CmH}_{2} \mathrm{O}$ & 99 & $\mathrm{CmH}_{2} \mathrm{O}$ \\
Predicho & 128 & $\mathrm{CmH}_{2} \mathrm{O}$ & 240 & $\mathrm{CmH}_{2} \mathrm{O}$ \\
$\%$ & 78 & & 41 & \\
\hline
\end{tabular}

Interpretación: $\quad \mathrm{Pi}_{\max }$ : Bajo (78\% del predicho)

$\mathrm{Pe}_{\max }$ : Bajo (41\% del predicho)

Figura 7: Se muestra ejemplo de informe de resultados.

\section{INTERPRETACIÓN}

Distintos grupos de investigación han informado valores normales de PRM en niños, adolescentes y adultos, ${ }^{13-16}$ y se ha observado una gran variabilidad en los puntos de corte o ecuaciones de referencia entre los diferentes grupos étnicos, lo cual podría resultar en falsos positivos y falsos negativos. Se considera que además de las diferencias entre los grupos étnicos, existen otros determinantes de los valores de las PRM como son la fuerza de los músculos respiratorios, la fuerza de retroceso elástico pulmonar, el crecimiento diferencial de las vías

Tabla 3: Ecuaciones de Black y Hyatt. ${ }^{15}$

\begin{tabular}{|c|c|c|}
\hline & Mujer & Hombre \\
\hline $\mathrm{Pi}_{\max }\left(\mathrm{cmH}_{2} \mathrm{O}\right)$ & $104-(0.51 \times$ edad $)$ & $143-(0.55 \times$ edad $)$ \\
\hline $\mathrm{Pe}_{\max }\left(\mathrm{cmH}_{2} \mathrm{O}\right)$ & $170-(0.53 \times$ edad $)$ & $268-(1.03 \times$ edad $)$ \\
\hline
\end{tabular}

Tabla 4: Ecuaciones de predicción para presiones respiratorias máximas en adultos (de 18 a 50 años) y en niños (7-17 años). ${ }^{13}$

\begin{tabular}{|l|c|c|}
\hline Grupo & $\mathrm{Pi}_{\max }\left(\mathrm{cmH}_{2} \mathrm{O}\right)$ & $\mathrm{Pe}_{\max }\left(\mathrm{cmH}_{2} \mathrm{O}\right)$ \\
\hline Hombres & $142-(1.03 \times \text { edad })^{\star}$ & $180-(0.91 \times \text { edad })^{\star}$ \\
\hline Mujeres & $-43+(0.71 \text { estatura })^{\ddagger}$ & $3.5+(0.55 \times \text { estatura })^{\ddagger}$ \\
\hline Niños & $44.5+(0.75 \times \text { peso })^{\S}$ & $35+(5.5 \times \text { edad })^{\star}$ \\
\hline Niñas & $40+(0.57 \times \text { peso })^{\S}$ & $24+(4.8 \times \text { edad })^{\star}$ \\
\hline
\end{tabular}

*Edad en años, 赫tatura en cm, ${ }^{\S}$ Peso en kg.
A

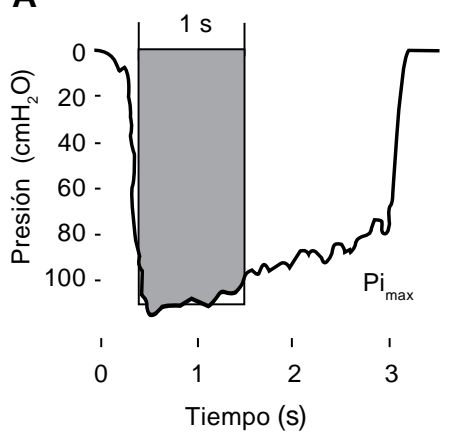

B

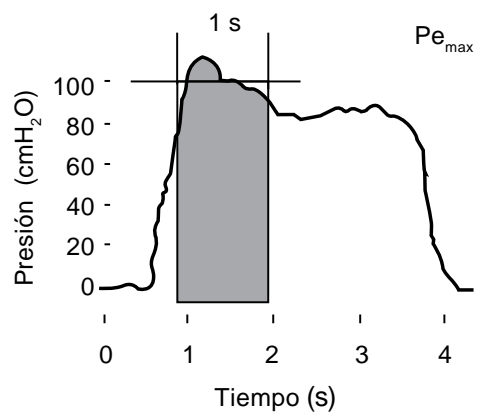

respiratorias, la distensibilidad de la pared torácica y las dimensiones del tórax. ${ }^{17}$ Otros aspectos importantes a considerar en los valores de referencia publicados son: el equipo utilizado, el tipo de boquilla, la posición para realizar la maniobra y las variables antropométricas incluidas en la ecuación.

De tal manera que la interpretación de los resultados debe ser cuidadosa y siempre debe realizarse tomando en cuenta la historia clínica, la patología del paciente y las condiciones fisiológicas que pueden ser determinantes en el momento de realizar el examen (hiperinflación o volúmenes pulmonares pequeños). ${ }^{12}$

En la interpretación deben tenerse en cuenta los porcentajes de los valores medidos con relación a las ecuaciones de referencia, considerándose normal las medidas $>80 \%$ del valor predicho o que se encuentren por arriba del límite inferior de la normalidad. .2,18 $^{2}$

Otra propuesta de interpretación es considerar puntos de corte en valores absolutos, un valor de $\mathrm{Pi}_{\text {max }}$ (medida a partir del volumen residual [VR]) igual o mayor a $75 \mathrm{cmH}_{2} \mathrm{O}$ para hombres y $50 \mathrm{cmH}_{2} \mathrm{O}$ para mujeres es considerado como «normal», y para la $\mathrm{Pe}_{\max }$ (medido a partir de la capacidad pulmonar total [CPT]) un valor igual o mayor de $100 \mathrm{cmH}_{2} \mathrm{O}$ para hombres y de $80 \mathrm{CmH}_{2} \mathrm{O}$ para mujeres ${ }^{15}$ (Tabla 3).

Otros valores de referencia propuestos se observan en la Tabla 4.

\section{CONCLUSIONES}

La medición de PRM es una prueba sencilla, de gran utilidad en la práctica de la medicina respiratoria; sin 
embargo, es dependiente del esfuerzo del paciente, por lo que, además de contar con equipos que cumplan con los estándares internacionales, se requiere ejecutarla de forma estandarizada y con adecuado control de calidad.

\section{REFERENCIAS}

1. Ruppel G. Manual of pulmonary function testing. 7th ed. St Louis, Missouri: Mosby; 1998.

2. Troosters T, Gosselink R, Decramer M, et al. Respiratory muscle assessment. Eur Respir Mon 2005;31:57-71.

3. Green M, Road J, Sieck GC, et al.; American Thoracic Society/ European Respiratory Society. ATS/ERS Statement on respiratory muscle testing. Am J Respir Crit Care Med 2002;166(4):518-624.

4. Vargas-Domínguez $C$, Mejía-Alfaro R, Martínez-Andrade R, SilvaCerón M, Vázquez-García JC, Torre-Bouscoulet L. Prueba de desaturación y titulación de oxígeno suplementario. Recomendaciones y procedimientos. Neumol Cir Torax 2009;68(4):162-173.

5. Gochicoa-Rangel L, Vázquez-García JC, Vargas-Domínguez C, et al. Prueba de reto bronquial con ejercicio. Recomendaciones y procedimiento. Neumol Cir Torax 2012;71(3):250-263.

6. Gochicoa-Rangel L, Cantú-González G, Miguel-Reyes JL, RodríguezMoreno L, Torre-Bouscoulet L. Oscilometría de impulso. Recomendaciones y procedimiento. Neumol Cir Torax 2014;73(2):138-149.

7. Heijdra YF, Dekhuijzen PN, van Herwaarden CL, Folgering HT. Effects of body position, hyperinflation, and blood gas tensions on maximal respiratory pressures in patients with chronic obstructive pulmonary disease. Thorax 1994;49(5):453-458.

8. Vincken W, Ghezzo H, Cosio MG. Maximal static respiratory pressures in adults: normal values and their relationship to determinants of respiratory function. Bull Eur Physiopathol Respir 1987;23(5):435-439.
9. Koulouris N, Mulvey DA, Laroche CM, Green M, Moxham J. Comparison of two different mouthpieces for the measurement of Pimax and Pemax in normal and weak subjects. Eur Respir $\mathrm{J}$ 1998;1(9):863-867.

10. Fiz JA, Texidó A, Izquierdo J, Ruiz J, Roig J, Morera J. Postural variation of the maximum inspiratory and expiratory pressures in normal subjects. Chest 1990;97(2):313-314.

11. O'Neill S, McCarthy DS. Postural relief of dyspnoea in severe chronic airflow limitation: relationship to respiratory muscle strength. Thorax 1983;38(8):595-600.

12. Evans JA, Whitelaw WA. The assessment of maximal respiratory mouth pressures in adults. Respir Care 2009;54(10):1348-1359.

13. Wilson SH, Cooke NT, Edwards RH, Spiro SG. Predicted normal values for maximal respiratory pressures in caucasian adults and children. Thorax 1984;39(7):535-538.

14. Gaultier C, Zinman R. Maximal static pressures in healthy children. Respir Physiol 1983;51(1):45-61.

15. Black LF, Hyatt RE. Maximal respiratory pressures: normal values and relationship to age and sex. Am Rev Respir Dis 1969:99(5):696-702.

16. Enright PL, Kronmal RA, Manolio TA, Schenker MB, Hyatt RE. Respiratory muscle strength in the elderly. Correlates and reference values. Cardiovascular Health Study Research Group. Am J Respir Crit Care Med 1994;149(2 Pt 1):430-438.

17. Johan A, Chan CC, Chia HP, Chan OY, Wang YT. Maximal respiratory pressures in adult Chinese, Malays and Indians. Eur Respir J 1997;10(12):2825-2828.

18. Hautmann $\mathrm{H}$, Hefele $\mathrm{S}$, Schotten $\mathrm{K}$, Huber RM. Maximal inspiratory mouth pressures (PIMAX) in healthy subject what is the lower limit of normal? Respir Med 2000;94(7):689-693.

Los autores declaran no tener conflicto de intereses. 